\title{
Assessment of circulating anti-Müllerian hormone in women using hormonal contraception: a systematic review
}

Saad Amer ${ }^{\mathrm{a}, *}$, Cathryn James ${ }^{\mathrm{b}}$, Tarek Khalf Al-Hussainic ${ }^{\mathrm{c}}$, Ahmed Aboelfadle Mohamed ${ }^{\mathrm{a}, \mathrm{c}}$

${ }^{a}$ Department of Obstetrics and Gynaecology, University of Nottingham, Royal Derby Hospital, Derby, United Kingdom, DE22 3DT.

${ }^{\mathrm{b}}$ Library \& Knowledge Service, Derby Teaching Hospitals NHS Foundation Trust, Derby, United Kingdom, DE22 3NE.

${ }^{c}$ Permanent address: Department of Obstetrics and Gynaecology, Assuit University, Asyut, Egypt.

Running title: Effect of hormonal contraction on circulating ant-Müllerian hormone

Funding was obtained from the Egyptian Cultural Centre and Education bureau in London and the British Council in Cairo.

\section{* Corresponding author:}

Saad A K S Amer, MD, FRCOG

Division of Medical Sciences \& Graduate Entry Medicine

School of Medicine

University of Nottingham

Royal Derby Hospital Centre

Uttoxeter Road

Derby DE22 3DT United Kingdom

Email: saad.amer@nottingham.ac.uk

Tel: +447957567635

Office: +44 1332786773 


\section{Abstract}

Background: The status of ovarian reserve markers during hormonal contraception (HC) remains uncertain with conflicting literature data. The purpose of this study was to assess the impact of hormonal contraception (HC) on circulating anti-Müllerian hormone (AMH) and other ovarian reserve markers. Methods: A systematic review was conducted including all cohort, cross-sectional and randomized controlled studies assessing serum anti Müllerian hormone concentration in women using hormonal contraception. Data sources included MEDLINE, EMBASE, Dynamed Plus, ScienceDirect, TRIP database, ClinicalTrials.gov and the Cochrane Library from January 2000 to October 2018. Results: A total of 366 studies were identified, of which 15 were eligible including 3280 women, mostly using combined hormonal contraception (CHC). Articles were divided according to duration of $\mathrm{HC}$ into short- (2-3 weeks), medium- (2-6 months), long- (>1year) and varied-term studies. Two study designs were identified including studies comparing AMH before and during/after CHC and studies comparing $\mathrm{CHC}$ users versus non-users. Short- and medium-term studies $(\mathrm{n}=284)$ reported no change in circulating AMH in women using cyclical $\mathrm{CHC}$ for 1-6 cycles. Apart from one study, all long- and varied-term studies (six studies, $\mathrm{n}=1601$ ) consistently showed a marked decline in AMH, antral follicle count (AFC) and ovarian volume $(\mathrm{OV})$. Three long-term studies $(\mathrm{n}=1324)$ provided evidence of AMH recovery after discontinuation of HC. Conclusion: circulating AMH seems to remain unchanged in women using cyclical CHC for up to six months, but appears to markedly decline in long-term users with recovery after discontinuation.

Registration number: PROSPERO (CRD42016046703).

Keywords: hormonal contraception, anti-Müllerian hormone, ovarian reserve, antral follicle count and ovarian volume. 


\section{Introduction}

Currently, an increasing number of women particularly in the developed world are on long-term hormonal contraception (HC) for various reasons such as education, career establishment, partnership changes and economic uncertainty. ${ }^{1}$ On the other hand, there has been a growing demand amongst young women, including those on long-term HC, to establish their fertility status and to assess their reproductive lifespan in order to help them plan their future. ${ }^{2,3}$ However, most long-term HC usurers would not want to risk a pregnancy by stopping their contraception for ovarian reserve assessment.

Commonly used ovarian reserve tests include biochemical markers such as circulating follicle stimulating hormone (FSH), inhibin B, oestradiol ( $\left.\mathrm{E}_{2}\right)$ and anti-Müllerian hormone (AMH) and ultrasound markers including antral follicle count (AFC) and ovarian volume (OV). Currently, circulating AMH is widely accepted as the most reliable biochemical ovarian reserve marker, which correlates well with the AFC. ${ }^{4}$

Oral combined hormonal contraception (CHC) is widely used as a safe, effective and reversible method. Its contraceptive effect is mainly achieved by suppression of the hypothalamo-pituitary-ovarian (HPO) axis, with subsequent inhibition of folliculogenesis and ovulation. ${ }^{5}$ The 7 -day hormone-free interval is usually associated with some recovery of the HPO axis, with resumption of follicle growth. ${ }^{6}$ It is possible that this recovery could maintain normal ovarian reserve as assessed by the number of small antral follicles and circulating antiMüllerian hormone (AMH). This hypothesis remains to be investigated.

Although, literature data are consistent on the inhibitory effect of $\mathrm{HC}$ on $\mathrm{FSH}$ and $\mathrm{E}_{2}$, data on $\mathrm{AMH}$ during $\mathrm{HC}$ are conflicting. According to earlier studies it was generally believed that circulating AMH acts as an autocrine/paracrine, rather than a systemic factor, which is not influenced by the hypothalamo-pituitary ovarian (HPO) axis. $^{7}$ This view was supported by several early studies confirming the stability of circulating AMH during different phases of the menstrual cycle. ${ }^{8-12}$ Based on this concept, it has been suggested that circulating AMH may not be affected by HC. More recent studies, however, revealed that AMH levels fluctuate considerably during the menstrual cycle and is not as stable as previously thought. ${ }^{13,14}$ 
A recent systematic review of endocrine and sonographic markers of ovarian function during HC highlighted the controversy over the status of AMH. ${ }^{15}$ However, the focus of that review was on ovarian morphological parameters with exclusion of the majority of studies assessing AMH. Whilst, some studies reported decline in circulating $\mathrm{AMH}$ in $\mathrm{HC}$ users, ${ }^{16-24}$ others found no change in $\mathrm{AMH} .{ }^{25-29}$

Based on the above, the status of circulating AMH as well as other ovarian reserve markers in HC users remains largely uncertain. We have therefore designed this systematic review to address this uncertainty.

\section{Methods}

This systematic review was designed in accordance with Preferred Reporting Items for Systematic Reviews and Meta-analyses (PRISMA) guidelines ${ }^{30}$ and was prospectively registered in PROSPERO (CRD42016046703).

We considered all published cohort (retrospective and prospective) and cross-sectional studies as well as randomized trials that investigated circulating $\mathrm{AMH}$ in women using $\mathrm{HC}$.

The primary outcome measure was serum AMH concentration and the secondary measures included AFC, OV, serum concentrations of FSH and $\mathrm{E}_{2}$

\section{Search strategy}

A search was conducted using various electronic databases from January 2000 to July 2017 (AMH was not in use before 2000). These databases included MEDLINE, EMBASE, Dynamed Plus, ScienceDirect, TRIP database, ClinicalTrials.gov and the Cochrane Library. Combinations of the following MeSH terms were used: hormonal contraception, oral contraception, combined contraception, contraceptive agents, intrauterine devices, Anti-Müllerian Hormone, Follicle Stimulating Hormone, ovarian follicle, ovarian reserve, and ovarian function tests. In addition to using MeSH terms search, title and abstract searches were also undertaken. Papers were limited to English Language, Human Studies and females. An accredited Clinical Librarian (CJ) performed the searches, which were then independently repeated by a second accredited Librarian using the same criteria. All identified articles were retrieved, and their reference lists were manually checked for further relevant studies. Published conference abstracts were also considered for inclusion. 


\section{Study selection}

Title and abstract of all identified articles were screened independently by two reviewers (AM, CJ) to assess relevance to this systematic review. In case of disagreement, the full text was retrieved and reviewed independently by a senior author (SA) for a final decision.

All identified articles were evaluated according to a standardized format including study design, methods, participant characteristics, intervention, and results. Two investigators (AM, SA) assessed eligibility of the studies independently. In case of discrepancies, a consensus was reached after discussion.

\section{Quality of included studies and risk of bias assessment}

Modified Newcastle-Ottawa tool was used to assess the quality and risk of bias of the included studies. ${ }^{31-33}$ Each article was scored according to three categories including selection (maximum three stars), comparability (four stars), and outcomes (two stars). Selection was rated according to recruitment bias, selection of consecutive participants and power calculation. Comparability was assessed based on adjustment of analysis for four confounders including patients' age $(<40)$, age-matching in cross-sectional and randomised studies, baseline serum AMH in cohort studies and the type and duration of hormonal contraception. Outcome was scored according to completeness of a follow-up period of at least three weeks for the short-term studies, three months for the medium-term studies and one year for the long-term studies. In the current analysis, we have given more weight to comparability factors and used the cut-off level of six stars with a minimum of three stars in the comparability category. Each article was scored initially by one author (AM) and then independantly reviewed and finalised by a second author (SA). All studies were included in the review with refernce to studies, which rate high on the quality assessment tool. Table 1 shows the results of quality scores of all studies in this review.

\section{Results}

\section{Characteristics of included Studies}

A total of 366 studies were identified through the electronic search, of which 347 did not measure AMH and were therefore excluded. The remaining 19 studies measured circulating AMH in $\mathrm{HC}$ users and were reviewed 
in full. Of these, two studies were excluded either due to lack of comparative AMH data ${ }^{34}$ or duplication. ${ }^{35}$ Tow further studies including women with PCOS were excluded. ${ }^{15,26}$ A total of 15 studies including $3280 \mathrm{HC}$ users were eligible for the review, of which, 13 measured circulating AMH during HC. ${ }^{16,18-27,29,36}$ The remaining two articles assessed AMH after HC discontinuation. ${ }^{37,38}$ Details of the 17 included studies are shown in Tables 2 and 3 .

Eight studies scored $\geq$ six on the modified Newcastle-Ottawa tool and were deemed of high quality. ${ }^{15,19,24-27,35}$

The high heterogeneity between the included studies precluded a quantitate synthesis of the data. Studies varied significantly in designs, statistical methods, HC types, duration of follow up and types of AMH assays. A narrative synthesis was therefore applied without meta-analysis.

\section{Study designs}

This review included four cohort studies, ${ }^{16,25,27,38}$ three RCTs, ${ }^{19,}$ 24, 34 and eight cross-sectional studies. ${ }^{8-20,22-24,29,37}$ In all RCTs, the arm including HC users with AMH data before and during/after HC was used as a cohort study for the purpose of this review.

Articles were divided into two main study designs including eight cohort/RCT studies comparing circulating AMH before and during HC (Table 2) and seven cross-sectional studies comparing AMH data between HC users versus non-users (Table 3).

\section{Types of hormonal contraception}

Three studies did not specify the HC (Tables 3). ${ }^{18,20,22}$ All remaining 12 studies used combined HC (CHC) either as the only HC (10 studies) $)^{16,19,21,23-26,29,36-38}$ or in addition to POC (two studies). ${ }^{23,27} \mathrm{HC}$ components for each study are presented in tables 2 and 3 . 


\section{Duration of hormonal contraception use}

The review included three short-term (2-3 weeks), ${ }^{16,26,36}$ three medium-term (2-6 months), ${ }^{21,25,27}$ four long-term $\left(>1\right.$ year) ${ }^{19,22,24,29}$ and three varied/unspecified-term ${ }^{18,20,23}$ studies. Three studies evaluated AMH after HC discontinuation in long-term users..$^{22,37,38}$

\section{Participants}

Majority of studies used appropriate inclusion and exclusion criteria. All short- and medium-term studies defined their participants as normo-ovulatory, ${ }^{16,21,25-28}$ with one study including a second group of PCOS users ${ }^{25}$ (excluded from the review) (Table 2). While most long- and varied-term studies included healthy women with no information on menstrual status before starting HC (Table 3). With regards to age, all studies included women in their twenties or thirties (Tables 2 and 3).

\section{$\underline{\text { AMH assays }}$}

$\underline{\text { Seven studies measured serum AMH concentrations using DSL enzyme-linked immunosorbent sensitive assay }}$ (ELISA) (DSL-10-14400, Diagnostic Systems Laboratories, Inc., Webster, USA). ${ }^{16,18,20,25,29,36,38}$ The reported $\underline{\text { limits for detection varied between } 0.006 \text { and } 0.1 \mathrm{ng} / \mathrm{mL} \text {, and the intra- and interassay coefficients of variation }}$ (CV) were 5.0 and $8.0 \%$, respectively.

Four studies assayed serum AMH levels using the IOT AMH ELISA kit (Immunotech-Beckman, Marseilles, France). ${ }^{19,24,26,27}$ The sensitivity of the assay is $0.7 \mathrm{pmol} / \mathrm{l}$, and the intra- and inter-assay coefficients of variation were $12.3 \%$ and $14.2 \%$, respectively.

Three studies analysed serum AMH concentrations using AMH Gen II ELISA (Diagnostic System Laboratories-Beckman Coulter Inc) with a detection limit of $0.08 \mathrm{ng} / \mathrm{mL} .^{21-23}$ The interassay and intra-assay coefficients of variation were $3.35 \%$ and $4.0 \%$, respectively.

One recent study measured serum AMH using the fully automated Access AMH assay (Beckman Coulter, Brea, CA) with $0.01 \mathrm{ng} / \mathrm{mL}$ limit of quantitation. ${ }^{37}$ 


\section{Circulating AMH and other ovarian reserve markers in CHC users}

\section{Short-term CHC users (2-3 weeks)}

Three studies ( $\mathrm{n}=243$ ) assessed circulating AMH in healthy short-term CHC users (Table 1). ${ }^{16,26,36}$ Two of these studies ( $n=223$ ) reported no change in circulating AMH after 2-3 weeks of CHC starting in the early follicular phase. ${ }^{26,36}$ The third study $(n=20)$ reported a decline in AMH after 2-3 weeks of COCP starting in mid-luteal phase ${ }^{16}$ The discrepancy between these results could be attributed to different timing of starting the COCP.

Only one small study conducted by Streuli and colleagues scored high on the quality assessment tool. ${ }^{26}$ This was one of the earlier studies assessing circulating AMH (using IOT) in 14 norm-ovulatory women before and after three weeks (cycle days 5-25) of CHC (oral pills, $n=7$; vaginal ring, $n=7$ ). Although this was an RCT, each arm was suitable for this review as a cohort study with $\mathrm{AMH}$ data before and after $\mathrm{CHC}$. The results showed no change in circulating AMH with either $\mathrm{CHC}$ forms. However, the authors stated that further studies were still needed to verify their findings.

Andersen et al. measured circulating AMH (using DSL), AFC and OV before and after 2-3 weeks of combined oral contraceptive pill (COCP) starting on cycle day 2-3 in 209 normo-ovulatory women scheduled for in-vitro fertilization (IVF). ${ }^{36}$ Measurements were performed on day 2-3 before and five days after the last COCP (before starting IVF). Although this was a multicentre RCT comparing IVF outcomes in women receiving COCP versus women receiving no $\mathrm{HC}$, we included the $\mathrm{COCP}$ arm as a cohort study where $\mathrm{AMH}$ data were reported before and after COCP. The results showed no change in circulating AMH (median $\left[5^{\text {th }}, 95^{\text {th }}\right.$ percentiles $] 2.2[0.5,6.3]$ versus $2.1[0.6,4.6] \mathrm{ng} / \mathrm{ml}), \mathrm{AFC}$ or OV.

Arbo et al. assessed circulating AMH (using DSL), AFC (2-10mm) and OV in 20 normo-ovulatory women before and after 2-3 weeks of COCP starting in midluteal phase (Table 2). ${ }^{16}$ Ovarian reserve was assessed on cycle day 3 before and after COCP (or 14 days after COCP in the absence bleeding). Circulating AMH significantly $(\mathrm{p}=0.04)$ decreased from median $[\mathrm{IQR}] 3.02 \mathrm{ng} / \mathrm{mL}[1.2,6.4]$ to $2.2 \mathrm{ng} / \mathrm{mL}[0.9,3.1]$ after COCP. There was no change in AFC, but the size of follicles and OV decreased. The authors concluded that AMH 
reduction was due to reduced production by the arrested follicles. The weakness of this study is its small size and the unusual timing of the $\mathrm{HC}$.

\section{Medium-term CHC users (2-6 months)}

Three studies assessed circulating AMH after 2-6 months of CHC in 103 women (Table 2). ${ }^{21,25,27}$ All three studies scored $\geq 6$ on the quality assessment scale. Two studies $(n=61)$ using cyclical $\mathrm{CHC}$ revealed no change of circulating $\mathrm{AMH}^{25,27}$ and one $(\mathrm{n}=42)$ using continuous COCP reported a decline in circulating $\mathrm{AMH} .{ }^{21}$

Somunkiran et al. assessed circulating AMH (using DSL), OV, AFC (2-9 mm), FSH and $\mathrm{E}_{2}$ before and after six months of cyclical COCP in 15 normo-ovulatory women. ${ }^{25}$ They reported no change in AMH $(1.93 \pm 0.51$ versus $2.11 \pm 0.56 \mathrm{ng} / \mathrm{ml}$ ). However, $\mathrm{OV}$, follicle number and size, FSH and $\mathrm{E}_{2}$ significantly decreased at six-months. The authors explained the lack of any change in AMH despite the decrease in small antral follicles by the continued production of AMH from smaller undetectable follicles. The final conclusion was that AMH may be considered as the only valid ovarian function marker in COCP users. However, given the small size of the study and the lack of sample size calculation, these results should be considered preliminary.

Li et al. measured circulating AMH (using Gen II) in 46 women before and after 3-4 months of cyclical CHC (COCP, $n=23$; injectable, $n=23) .{ }^{27}$ They reported no change in circulating AMH in either of the groups. Pretreatment median (IQR) circulating AMH were 27.2 (26-35) pmol/l for COCP and 20.9 (13.2-35.4) pmol/l for injectable CHC users. Post-treatment values were 17.1 (8.5-28.6) and 26.9 (9.0-44.0) for COCP and injectable CHC respectively. Although the study was powered (90\%) to detect a difference of 1 SD of AMH, the authors did not clarify what that SD was. It seems that this SD was large and the study was not powered to detect smaller but clinically important differences. This is supported by the obvious trend towards a large drop of circulating AMH in COCP users from 27.2 to $17.1 \mathrm{pmol} / \mathrm{L}$. The lack of statistical significance here could purely be due to the small sample size. Other limitations of this study include the variation of timing of AMH measurement and the lack of any information on the menstrual status of the participants and whether they included PCOS women. 


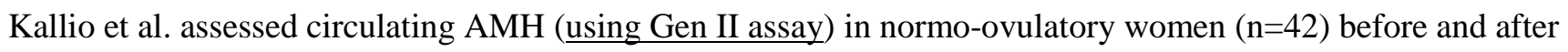
five and nine weeks of continuous $\mathrm{CHC}$ including $\mathrm{COCP}(\mathrm{n}=13)$, transdermal patches $(\mathrm{n}=15)$ and vaginal rings $(\mathrm{n}=14) .{ }^{21}$ They reported a significant $(\mathrm{P}<.001)$ decline in circulating AMH with all CHC forms after nine weeks (COCP, $3.9 \pm 3.0$ vs. $1.9 \pm 1.5 \mathrm{ng} / \mathrm{Ml}$; transdermal, $3.9 \pm 3.6$ vs. $2.0 \pm 1.3 \mathrm{ng} / \mathrm{mL}$; vaginal ring, $4.3 \pm 3.5$ vs. $2.3 \pm 1.2$ $\mathrm{ng} / \mathrm{mL})$. Similar significant $(\mathrm{P}<.001)$ declines were observed in circulating $\mathrm{FSH}$, inhibin $\mathrm{B}$ and $\mathrm{E}_{2}$ in all groups at five and nine weeks of treatment. The authors hypothesised that the decline of circulating AMH could be due to reduced follicular granulosa cell mass as a result of the CHC-induced FSH suppression. However, it is known that the earlier follicular growth is independent of FSH. ${ }^{39}$ The main limitation of this study, is its small size and the lack of power calculation.

\section{Long-term CHC users (>1 year)}

Four cross-sectional studies investigated ovarian reserve in long-term users ( $\mathrm{n}=1414)$ of CHC versus non-users $(\mathrm{n}=2273)$ (Table 3). ${ }^{19,22,24,29}$ Three studies reported lower circulating AMH in CHC users ( $\left.\mathrm{n}=1380\right)$ compared to non-users $(\mathrm{n}=2237) .{ }^{19,22,24}$ The remaining study showed no difference in circulating AMH in CHC users $(\mathrm{n}=34)$ versus non-users $(n=36) .{ }^{29}$

With regards to AFC, two studies showed reduction in the number of larger follicles, but no change in the number of smaller follicles measuring $2-4 \mathrm{~mm}(22)$ or $2-6 \mathrm{~mm} .{ }^{29}$ The third study reported lower AFC of all follicle size categories in CHC users. ${ }^{19}$

Only one study in this group rated high on the quality assessment scale. ${ }^{29}$ This was a well-designed crosssectional study assessing circulating AMH (using DSL assay), AFC and OV in 34 healthy women using COCP for >1year (average (range) 3.16 (2.4-6.0) Years) and 36 age-matched healthy non-HC users. Assessment was carried out in the early follicular phase in both groups. They found no differences in circulating AMH or the number of the $2-6 \mathrm{~mm}$ follicles between the two groups. While, follicles measuring $>6 \mathrm{~mm}$ and OV were significantly $(\mathrm{P}<0.001)$ lower in COCP users versus non-users. Circulating $\mathrm{FSH}, \mathrm{LH}$ and $\mathrm{E}_{2}$ were significantly $(\mathrm{P}<0.05)$ lower in the COCP group. They concluded that prolonged use of COCP suppresses pituitary gonadotropins and antral follicle development beyond $6 \mathrm{~mm}$, but had no effect on circulating AMH or the 
number of small antral follicles. The main limitation of this study was the lack of power calculation for AMH as the sample size was calculated for AFC as the primary outcome.

Bentzen et al. compared early follicular (day 2-5 or pill free interval) circulating AMH (using IOT assay), AFC and OV among $228 \mathrm{CHC}$ users (COCP, n=217; vaginal rings, $n=11$ ) versus 504 non-users. ${ }^{19} \mathrm{COCP}$ included monophasic with $20 \mu \mathrm{g} E \mathrm{E}(\mathrm{n}=101)$, monophasic with $30-35 \mu \mathrm{g} E \mathrm{E}(\mathrm{n}=96)$ and bi-/triphasic preparations $(\mathrm{n}=20)$. After adjusting for age, they reported 30\% (95\%CI, 19.9-38.5\%) lower AMH, 30\% (95\%CI, 23.6-36.7\%) lower AFC (all follicle sizes) and 42\% (95\%CI, 37.8-46.3\%) lower OV in CHC users versus non-users. They also found a trend of negative linear association between duration of $\mathrm{CHC}$ and ovarian reserve parameters. They calculated a decline of $2.3 \%$ in AMH (not statistically significant), $2.5 \%$ of $\mathrm{AFC}(\mathrm{P}=0.02)$ and $2.7 \%$ of OV $(\mathrm{p}=0.005)$ for every year of CHC. No dose-response relation was found between the dose of EE and AMH or AFC. They concluded that circulating AMH and AFC are lowered in a duration-, but not dose-dependent fashion in COCP users and that these makers may not be reliable in assessing ovarian reserve in these women.

In a large population-based cross-sectional study, Dolleman et al. investigated the effect of various reproductive and lifestyle factors including oral $\mathrm{HC}$ on circulating AMH (using Gen II assay). ${ }^{22}$ The HC type was not specified and its duration varied widely ( $>1$ to $>20$ years). They calculated age-specific AMH percentiles using general linear modelling with CG-LMS (Cole and Green, Lambda, Mu, and Sigma model). They reported that current oral HC users $(n=908)$ had an age-specific AMH of 11 percentiles lower $(\mathrm{P}<.0001)$ than non-users $(n=1090)$. They found no significant effect of HC duration on AMH in women not exceeding 20 years of use. Women who used $\mathrm{HC}>20$ years had an 8.8 \pm 4.2 percentiles lower age-specific AMH compared to women who used $\mathrm{HC}$ for $\leq 1$ year.

In a large cross-sectional study including 887 women aged 19-46 attending for fertility assessment, Petersen et al. compared circulating AMH (using IOT assay), AFC and OV in long-term CHC users (n=244) versus nonusers $(\mathrm{n}=643) .{ }^{24} \mathrm{CHC}$ included $\mathrm{COCP}(\mathrm{n}=225)$ and vaginal rings $(\mathrm{n}=19)$. The median HC duration was 12 years (90\% population limit; 4.0, 18.4 years). After adjusting for age by linear regression analysis, they found a $19 \%$ lower AMH (P<0.001, 95\%CI, 9.1\%, 29.3\%), 18\% lower AFC (95\% CI 11.2-24.8\%) and 50\% lower OV (95\% 
CI 45.1-53.7\%) in CHC users versus non-users. Notably, the decline in AFC was only observed in the 5-10mm follicles, but the 2-4mm follicles increased in $\mathrm{CHC}$ users. $\mathrm{HC}$ users and non-users were comparable regarding age, BMI, smoking and maternal age at menopause. They concluded that $\mathrm{CHC}$ seem to have a major negative impact on the $\mathrm{OV}$, and a moderate impact on $\mathrm{AFC}$ and $\mathrm{AMH}$. One weakness in this study is the inclusion of self-referred women attending the Fertility Assessment Clinic, which may not reflect the general population. Further, the timing of AMH assessment varied in different women, which could be a confounding factor.

\section{CHC users for varied/unspecified duration}

Three cross-sectional studies investigated ovarian reserve in HC users $(n=202)$ versus non-users $(n=312)$, but did not specify the duration of HC. ${ }^{18,20,23}$ All studies reported lower circulating AMH in HC users compared to non-users (Table 2). The three studies were considered of low quality with a low score on the modified Newcastle-Ottawa tool.

In a small cross-sectional study Shaw et al. reported significantly ( $\mathrm{P}<0.001)$ lower median AMH (using DSL) level (3.0 pmol/L (95\% CI, 2.1-3.8 pmol/L) in users of oral HC (n=14) compared to that (4.6 (95\% CI, 3.9-5.2) $\mathrm{pmol} / \mathrm{L})$ of non-users $(\mathrm{n}=121) .{ }^{18}$ The results were adjusted for age and all women were aged 33-45 years. Unfortunately, neither the type nor the duration of $\mathrm{HC}$ was specified in this study. Furthermore, timing of AMH measurement in the cycle was not mentioned.

In a population-based cross-sectional study, Kristensen et al. assessed circulating AMH (using DSL) in 180 HC users versus 76 non-users. ${ }^{20} \mathrm{HC}$ included $\mathrm{COCP}(\mathrm{n}=161)$, combined vaginal rings $(\mathrm{n}=12)$, progestogen only contraceptives (POC, $\mathrm{n}=7$ ). Unfortunately, the duration of $\mathrm{HC}$ was not specified in this study and AMH measurement was taken at varied times of the menstrual cycle. Although the focus of this study was mainly to study the association between circulating $\mathrm{AMH}$ and $\mathrm{AFC}$, androgens, and menstrual cycle characteristics in $\mathrm{HC}$ users versus non-users, AMH data in HC-users versus non-users were also presented and were considered relevant to this review. Circulating AMH in HC users (Median (IQR) 2.5 (1.5-3.5) ng/mL were significantly $(\mathrm{P}=0.01)$ lower than that $(2.9(1.8-4.3) \mathrm{ng} / \mathrm{mL})$ in non-users. The study also found a strong positive association between circulating $\mathrm{AMH}$ and androgen levels in non-users of $\mathrm{HC}$, but not in $\mathrm{HC}$ users. On the other hand, a 
strong relationship between AMH and AFC was observed in both users and nonusers. Analysis after exclusion of the seven POC users did not change the above results.

Johnson et al. compared circulating AMH (using Gen II) and AFC in HC-users ( $\mathrm{n}=50)$ including cancer survivors ( $\mathrm{n}=42)$ and healthy controls $(\mathrm{n}=8)$ versus non-users $(\mathrm{n}=189)$ including cancer survivors $(\mathrm{n}=84)$ and healthy controls $(n=115) .{ }^{23}$ For the purpose of this review and to avoid confounding effect of chemotherapy on ovarian reserve, we included only the healthy HC users $(n=8)$ and non-users $(n=115)$. After adjusting for confounders, AMH was 55\% lower among HC users (geometric mean (95\% CI) $1.02(0.67-1.56)$ ng/ml, $\mathrm{p}<0.001)$ versus non-users $(2.31(2.02-2.63) \mathrm{ng} / \mathrm{ml})$. AFC was $20 \%$ lower among healthy CHC users versus non-users (RR 0.80, 95\% CI 0.69-0.93). HC was COCP in most users, but exact numbers were not presented. $\mathrm{AMH}$ and $\mathrm{AFC}$ were assessed in the early follicular phase in the majority of cases. Although this study was described as a longitudinal cohort study, data used in this review were taken at one point and it is therefore considered a cross-sectional design. The weakness of this study is its small size. Furthermore, HC users were defined as women who used $\mathrm{HC}$ within the preceding three months of ovarian reserve assessment, meaning that some $\mathrm{HC}$ users were non-users at the time of assessment.

\section{AMH after CHC discontinuation in long-term users}

Three studies evaluated ovarian reserve after discontinuation of HC in long-term users ( $n=1324)$, of which two were cross-sectional studies assessing AMH in previous users versus women who had never used HC (Table 3). ${ }^{22,37}$ The third study was a cohort study comparing AMH during and after HC discontinuation. ${ }^{38}$ All studies suggested that the reduction of circulating AMH observed in long-term HC users seem to be reversable after discontinuation.

Two studies scored $\geq 6$ on the quality assessment tool, ${ }^{37,38}$ while the remaining study scored low. ${ }^{22}$

Kucera et al. compared circulating AMH (using Access AMH assay (Beckman Coulter, Brea, CA) in 105 previous long-term COCP users (one year after discontinuation) versus 44 women who had never used HC. ${ }^{37}$ The mean HC duration was 11.4 (range, 10-17) years. They reported no significant $(\mathrm{p}=0.326)$ difference 
between previous HC users (median (IQR), $2.89(1.57,4.46) \mathrm{ng} / \mathrm{ml})$ and women who had never used HC (3.37 $(2.07,4.42) \mathrm{ng} / \mathrm{ml})$. They concluded that there was no negative impact of previous $\mathrm{HC}$ use on circulating AMH.

In their large cross-sectional study, Dolleman et al. compared circulating AMH (measured with Gen II assay) in previous HC users ( $\mathrm{n}=1194)$ versus healthy controls who had never used HC. They reported that that previous $\mathrm{HC}$ use was not associated with low AMH values. ${ }^{22}$

Van den Berg et al. measured circulating AMH (using DSL assay), AFC and OV during CHC (on day 7 of the hormone-free interval (D7-HFI)) and in two subsequent natural cycles after CHC discontinuation in 25 users (median (IQR) duration, 20(57) months). ${ }^{38} \mathrm{CHC}$ included COCP (n=23, mono-/triphasic 20-50 $\mu \mathrm{g}$ oestrogen preparations), patch $(\mathrm{n}=1)$ and vaginal ring $(\mathrm{n}=1)$. They reported that D7-HFI AMH (mean $\pm \mathrm{sd}, 2.0 \pm 1.2 \mathrm{ng} / \mathrm{ml})$ remained unchanged in natural cycle-1 $(2.0 \pm 1.3 \mathrm{ng} / \mathrm{ml})$, but significantly $(\mathrm{p}<0.001)$ increased to $2.6 \pm 1.3 \mathrm{ng} / \mathrm{ml}$ in the natural cycle-2. AFC and OV increased after HC discontinuation from the D7-HFI to natural cycle-1 and from natural cycle-1 to cycle-2. The authors also reported that FSH, AMH and AFC values measured on D7HFI could be used to predict values of natural cycle- 2 after HC discontinuation using calculated equations, which require validation. ${ }^{38}$

\section{Circulating AMH in users of progestogen only contraception}

Only two studies investigated circulating AMH in a small number of users of progestogen only contraception (POC). ${ }^{23,27}$ Of these only the study by Li et al. provided AMH data separately for 49 POC users including POP $(n=9)$, POI $(n=20)$ and Mirena $(n=20) .{ }^{27}$ The results showed that pre-treatment serum AMH levels remained unchanged after 3-4 months of POC. The remaining study did not provide separate analysis for the POC users. ${ }^{23}$

\section{Discussion}

To the best of our knowledge, this is the first systematic review to investigate the status of circulating AMH and other ovarian reserve markers in a large number of HC users $(n=3440)$. We have found two main types of study designs addressing this issue. The first type included short- (2-3 weeks) and medium- (2-6 months) term cohort studies comparing AMH data before versus during/after HC. The second type included long- (>1year) and 
varied-term cross-sectional studies comparing HC users versus non-users. The limited data from short- and medium-term studies involving healthy non-PCOS women showed no change in circulating AMH during cyclical CHC. On the other hand, AMH was reduced after nine weeks of continuous CHC. ${ }^{21}$

Apart from one publication, ${ }^{29}$ all long-term studies as well as studies with unspecified/varied HC duration have consistently showed a markedly lower circulating AMH, AFC and OV in HC users versus non-users. The magnitude of $\mathrm{AMH}$ decline in long-term $\mathrm{HC}$ users varied widely from 14 to 55\%. Three long-term studies provided evidence for AMH recovery after discontinuation of HC. Only one relatively small, but well-designed study by Deb et al. showed no change of AMH in 34 CHC users. ${ }^{29}$ The discrepancy between this study and the other long-term studies could be attributed to differences in the study population and/or methodology. Whilst Deb and colleagues ${ }^{27}$ measured serum AMH in the early follicular phase, Petersen and colleagues ${ }^{24}$ measured it at a random time of the cycle and Panidis and colleagues ${ }^{28}$ measured it in mid-follicular phase (day 5-7). Furthermore, Deb et al. compared the median (range) AMH in users versus non-users, ${ }^{29}$ while several studies estimated the percentage difference in AMH or the AMH percentiles. Moreover, the age of women in the study by Deb et al. was much younger $(25.4 \pm 4.2$ years $)$ compared to most other studies. ${ }^{29}$

With regards to AFC and OV, two short-term studies showed no change in AFC, ${ }^{16,36}$ but one study showed a reduction in the follicle size and OV. ${ }^{16}$ Two medium-term studies showed a decrease in OV and AFC in PCOS $(15,23)$ and non-PCOS users of $\mathrm{CHC} .{ }^{25}$ Several long-term studies consistently reported a decline in AFC affecting either all follicle sizes ${ }^{19,23}$ or only large follicles. ${ }^{24,29}$ Several studies have reported reduction in OV. Two studies reported recovery of AFC and OV after HC discontinuation. ${ }^{37,38}$

Data on the effect of HC duration on AMH in long-term users are limited and conflicting. One study found a negative linear association between duration of $\mathrm{CHC}$ and ovarian reserve parameters, ${ }^{19}$ while another larger study reported no effect of $\mathrm{HC}$ duration on the magnitude of AMH decline, except in women exceeding 20 years of HC use. ${ }^{22}$

The majority of studies used COCP with very few using either other combined forms such as patches, vaginal rings or injectables or progestogen only preparations. There is hardly any data on the effect of different EE 
doses or different progestogen components. Also, only limited data are available on the effect of progestogen only preparations showing no effect on circulating AMH.

The wide variation in the magnitude of AMH decline (14-55\%) in long-term studies could be attributed to the variations in duration and types of $\mathrm{HC}$, populations investigated and the methodologies applied.

The observed decline in circulating FSH and $\mathrm{E}_{2}$ in most studies was expected and could be explained by HPOaxis suppression via a negative feedback mechanism. However, the decline of circulating AMH in long-term $\mathrm{HC}$ users is somewhat surprising as it has long been believed that $\mathrm{AMH}$ is an autocrine/paracrine factor, which is not influenced by the HPO-axis. ${ }^{8-12}$ However, as mentioned in the introduction, more recent studies revealed that circulating AMH fluctuates considerably during the menstrual cycle and may therefore be influenced by the HPO-axis. ${ }^{13,14}$ It is therefore possible that the decline in AMH in long-term HC users is due to HPO-axis suppression.

Based on the findings of this review, circulating AMH seems to be markedly supressed in long-term CHC users and cannot therefore be used for the accurate assessment of ovarian reserve in these women. Furthermore, AMH levels in long-term users cannot be used to calculate or predict the normal values due to the uncertainty about the magnitude of its decline. We therefore recommend that if assessment of ovarian reserve is required in chronic $\mathrm{HC}$ users, the $\mathrm{HC}$ should be discontinued for a washout period before measuring $\mathrm{AMH}$ and other markers. The length of this washout period requires further research to estimate the time required for the full recovery of circulating $\mathrm{AMH}$.

Apart from one study, which used the fully automated Access AMH assay, ${ }^{38}$ all studies included in this review used either the DSL, IOT or GEN II ELISA AMH assay kits. It is now well established that these kits, which are $\underline{\text { no longer in use, gave varied results and had different sensitivities and inter- and inter-assay coefficients of }}$ variation. The IOT assay (Immunotech, Beckman Coulter, Marseille, France) has been found to produce AMH concentrations 40\% higher compared with the DSL assay (Diagnostic Systems Laboratories), making it difficult to combine/compare results from different Studies. ${ }^{40}$ Gen II assay, which was first introduced by the merging of DSL and IOT companies, was calibrated to the old IOT standards and AMH levels were thus comparable with 
the IOT assay (41-43). The Gen II assay suffered significant interference problems until July 2013 and was later modified to overcome this issue. ${ }^{44}$ Furthermore, inter-laboratory variations and sample instability further complicate the interpretation and clinical implications of AMH values. ${ }^{44}$ Moreover, studies using AMH kits that produced low values (e.g. DSL) may not be able to detect differences in AMH levels especially in pobulations with low AMH levels.

Although this systematic review has consistently shown marked decline in circulating AMH in long-term HC users, these data should be interpreted with caution in view of the significant variations and high heterogeneity between the reviewed studies. Further well designed and sufficiently powered cohort studies are required to assess the short (<3months), medium (<1year) and long-term (>1year) status of circulating AMH during HC compared with pre-treatment baseline levels. This will be the appropriate design as it allows assessment of any changes in AMH in the same participant rather than comparing between users versus non-users, where several confounders could compromise the validity of the results. Based on this review, we suggest that any planned study should be powered to detect a 19-29\% decline in serum AMH concentration after $>1$ year of HC. In addition to a robust design, future studies should use the most sensitive and widely used automated AMH assays. Furthermore, future studies should focus on one form of $\mathrm{HC}$ in each study.

In conclusion, circulating $\mathrm{AMH}, \mathrm{AFC}$ and ovarian volume seem to decline markedly in long-term $\mathrm{HC}$ users. Short- and medium-term cyclical CHC does not seem to affect AMH in normo-ovulatory women. However, all these data should be considered preliminary given the poor quality and/or the small size of almost all the studies. The effect of HC on AMH in PCOS women remains uncertain. We recommend that further welldesigned studies are required to validate the above findings. 
References

1. Mills M, Rindfuss RR, McDonald P, Te Velde E. Why do people postpone parenthood? Reasons and social policy incentives. Hum Reprod Update. 2011;17:848-860.

2. Tremellen K, Savulescu J. Ovarian reserve screening: a scientific and ethical analysis. Hum Reprod. 2014;29:2606-2614.

3. Hvidman HW, Petersen KB, Larsen EC, Macklon KT, Pinborg A, Nyboe Andersen A. Individual fertility assessment and pro-fertility counselling; should this be offered to women and men of reproductive age?. Hum Reprod. 2014;30:9-15.

4. La Marca A, Sighinolfi G, Giulini S, et al. Normal serum concentrations of anti-Müllerian hormone in women with regular menstrual cycles. Reprod Biomed Online. 2010;21:463-469.

5. ESHRE Capri Workshop Group. Ovarian and endometrial function during hormonal contraception. Hum Reprod. 2001;16:1527-1535.

6. Schlaff WD, Lynch AM, Hughes HD, Cedars MI, Smith DL. Manipulation of the pill-free interval in oral contraceptive pill users: The effect on follicular suppression. Am J Obstet Gynecol. 2004;190:943-951.

7. Fanchin R, Schonäuer LM, Righini C, Guibourdenche J, Frydman R, Taieb J. Serum anti-Müllerian hormone is more strongly related to ovarian follicular status than serum inhibin B, estradiol, FSH and LH on day 3. Hum Reprod. 2003;18:323-327.

8. La Marca A, Stabile G, Artenisio AC, Volpe A. Serum anti-Mullerian hormone throughout the human menstrual cycle. Hum Reprod. 2006;21:3103-3107.

9. Hehenkamp WJ, Looman CW, Themmen AP, de Jong FH, Te Velde ER, Broekmans FJ. Anti-Mullerian hormone levels in the spontaneous menstrual cycle do not show substantial fluctuation. J Clin Endocrinol Metab. 2006;91:4057-4063.

10. Tsepelidis S, Devreker F, Demeestere I, Flahaut A, Gervy C, Englert Y. Stable serum levels of antiMüllerian hormone during the menstrual cycle: a prospective study in normo-ovulatory women. Hum Reprod. 2007;22:1837-1840. 
11. van Disseldorp J, Lambalk CB, Kwee J, et al. Comparison of inter-and intra-cycle variability of antiMüllerian hormone and antral follicle counts. Hum Reprod. 2009;25:221-227.

12. Dewailly D, Andersen CY, Balen A, et al. The physiology and clinical utility of anti-Müllerian hormone in women. Hum Reprod Update. 2014;20:370-385.

13. Overbeek A, Broekmans FJ, Hehenkamp WJ, et al. Intra-cycle fluctuations of anti-Müllerian hormone in normal women with a regular cycle: a re-analysis. Reprod Biomed Online. 2012;24:664-669.

14. Hadlow N, Longhurst K, McClements A, Natalwala J, Brown SJ, Matson PL. Variation in antimüllerian hormone concentration during the menstrual cycle may change the clinical classification of the ovarian response. Fertil Steril. 2013;99:1791-1797.

15. D'Arpe S, Di Feliciantonio M, Candelieri M, Franceschetti S, Piccioni MG, Bastianelli C. Ovarian function during hormonal contraception assessed by endocrine and sonographic markers: a systematic review. Reprod Biomed Online. 2016;33:436-448.

16. Arbo E, Vetori DV, Jimenez MF, Freitas FM, Lemos N, Cunha-Filho JS. Serum anti-müllerian hormone levels and follicular cohort characteristics after pituitary suppression in the late luteal phase with oral contraceptive pills. Hum Reprod. 2007;22:3192-3196.

17. Fábregues F, Castelo-Branco C, Carmona F, Guimerá M, Casamitjana R, Balasch J. The effect of different hormone therapies on anti-müllerian hormone serum levels in anovulatory women of reproductive age. Gynecol Endocrinol. 2011;27:216-224.

18. Shaw CM, Stanczyk FZ, Egleston BL, et al. Serum antimüllerian hormone in healthy premenopausal women. Fertil Steril. 2011;95:2718-2721.

19. Bentzen JG, Forman JL, Pinborg A, et al. Ovarian reserve parameters: a comparison between users and non-users of hormonal contraception. Reprod Biomed Online. 2012;25:612-619. 
20. Kristensen SL, Ramlau-Hansen CH, Andersen CY, et al. The association between circulating levels of antimüllerian hormone and follicle number, androgens, and menstrual cycle characteristics in young women. Fertil Steril. 2012;97:779-785.

21. Kallio S, Puurunen J, Ruokonen A, Vaskivuo T, Piltonen T, Tapanainen JS. Antimüllerian hormone levels decrease in women using combined contraception independently of administration route. Fertil Steril. 2013;99:1305-1310.

22. Dolleman M, Verschuren WM, Eijkemans MJ, et al. Reproductive and lifestyle determinants of antiMüllerian hormone in a large population-based study. J Clin Endocrinol Meta. 2013;98:2106-2115.

23. Johnson LN, Sammel MD, Dillon KE, Lechtenberg L, Schanne A, Gracia CR. Antimüllerian hormone and antral follicle count are lower in female cancer survivors and healthy women taking hormonal contraception. Fertil Steril. 2014;102:77-781.

24. Petersen KB, Hvidman HW, Forman JL, et al. Ovarian reserve assessment in users of oral contraception seeking fertility advice on their reproductive lifespan. Hum Reprod. 2015;30:2364-2375.

25. Somunkiran A, Yavuz T, Yucel O, Ozdemir I. Anti-Müllerian hormone levels during hormonal contraception in women with polycystic ovary syndrome. Eur J Obstet Gynecol Reprod Biol. 2007;134:196-201.

26. Streuli I, Fraisse T, Pillet C, Ibecheole V, Bischof P, De Ziegler D. Serum antimüllerian hormone levels remain stable throughout the menstrual cycle and after oral or vaginal administration of synthetic sex steroids. Fertil Steril. 2008;90:395-400.

27. Li HW, Wong CY, Yeung WS, Ho PC, Ng EH. Serum anti-müllerian hormone level is not altered in women using hormonal contraceptives. Contraception. 2011;83:582-585. 
28. Panidis D, Georgopoulos NA, Piouka A, et al. The impact of oral contraceptives and metformin on antiMüllerian hormone serum levels in women with polycystic ovary syndrome and biochemical hyperandrogenemia. Gynecol Endocrinol. 2011;27:587-592.

29. Deb S, Campbell BK, Pincott-Allen C, Clewes JS, Cumberpatch G, Raine-Fenning NJ. Quantifying effect of combined oral contraceptive pill on functional ovarian reserve as measured by serum anti-Müllerian hormone and small antral follicle count using three-dimensional ultrasound. Ultrasound Obstet Gynecol. 2012;39:574-580.

30. Liberati A, Altman DG, Tetzlaff J, et al. The PRISMA statement for reporting systematic reviews and meta-analyses of studies that evaluate health care interventions: explanation and elaboration. PLoS Med. 2009;6:e1000100.

31. Wells GA, Shea B, O’Connell D, et al. The Newcastle-Ottawa Scale (NOS) for assessing the quality of non-randomized studies in meta-analysis. Ottawa Health Research Institute. http://www.ohri.ca/programs/clinical_epidemiology/oxford.asp

32. Raffi F, Metwally M, Amer S. The impact of excision of ovarian endometrioma on ovarian reserve: a systematic review and meta-analysis. J Clin Endocrinol Metab. 2012;97:3146-3154.

33. Mohamed AA, Al-Hussaini TK, Fathalla MM, El Shamy TT, Abdelaal II, Amer SA. The impact of excision of benign nonendometriotic ovarian cysts on ovarian reserve: a systematic review. Amer J Obstet Gynecol. 2016;215:169-176.

34. Steiner AZ, Stanczyk FZ, Patel S, Edelman A. Antimullerian hormone and obesity: insights in oral contraceptive users. Contraception. 2010;81:245-248.

35. Bentzen JG, Forman JL, Larsen EC, et al. Maternal menopause as a predictor of anti-Müllerian hormone level and antral follicle count in daughters during reproductive age. Hum Reprod. 2012;28:247-255.

36. Andersen AN, Witjes H, Gordon K, Mannaerts B. Predictive factors of ovarian response and clinical outcome after IVF/ICSI following a rFSH/GnRH antagonist protocol with or without oral contraceptive pre-treatment. Hum Reprod. 2011;26:3413-3423. 
37. Kucera R, Ulcova-Gallova Z, Topolcan O. Effect of long-term using of hormonal contraception on antiMüllerian hormone secretion. Gynecol Endocrinol. 2016;32:383-385.

38. Van den Berg MH, Van Dulmen-den Broeder E, Overbeek A, et al. Comparison of ovarian function markers in users of hormonal contraceptives during the hormone-free interval and subsequent natural early follicular phases. Hum Reprod. 2010;25:1520-1527.

39. Barnes RB, Namnoum AB, Rosenfield RL, Layman LC. The role of LH and FSH in ovarian androgen secretion and ovarian follicular development: clinical studies in a patient with isolated FSH deficiency and multicystic ovaries: case report. Hum Reprod. 2002;17:88-91.

40. Fréour T, Mirallié S, Bach-Ngohou K, Denis M, Barrière P, Masson D. Measurement of serum anti-Müllerian hormone by Beckman Coulter ELISA and DSL ELISA: comparison and relevance in assisted reproduction technology (ART). Clin Chim Acta. 2007;375:162-164.

41. Broer SL, Broekmans FJ, Laven JS, Fauser BC. Anti-Müllerian hormone: ovarian reserve testing and its potential clinical implications. Hum Reprod Update. 2014;20:688-701.

42. Kumar A, Kalra B, Patel A, McDavid L, Roudebush WE. Development of a second generation antiMüllerian hormone (AMH) ELISA. J Immunol Methods. 2010;362:51-59.

43. Wallace AM, Faye SA, Fleming R, Nelson SM. A multicentre evaluation of the new Beckman Coulter antiMullerian hormone immunoassay (AMH Gen II). Ann Clin Biochem. 2011;48:370-373.

44. Magnusson $\AA$ A, Oleröd G, Thurin-Kjellberg A, Bergh C. The correlation between AMH assays differs depending on actual AMH levels. Hum Reprod Open. 2017 8;2017:1-5. 
Figure captions

Figure 1. Preferred Reporting Items of Systematic Reviews and Meta-Analyses (PRISMA) flow chart of the study selection process
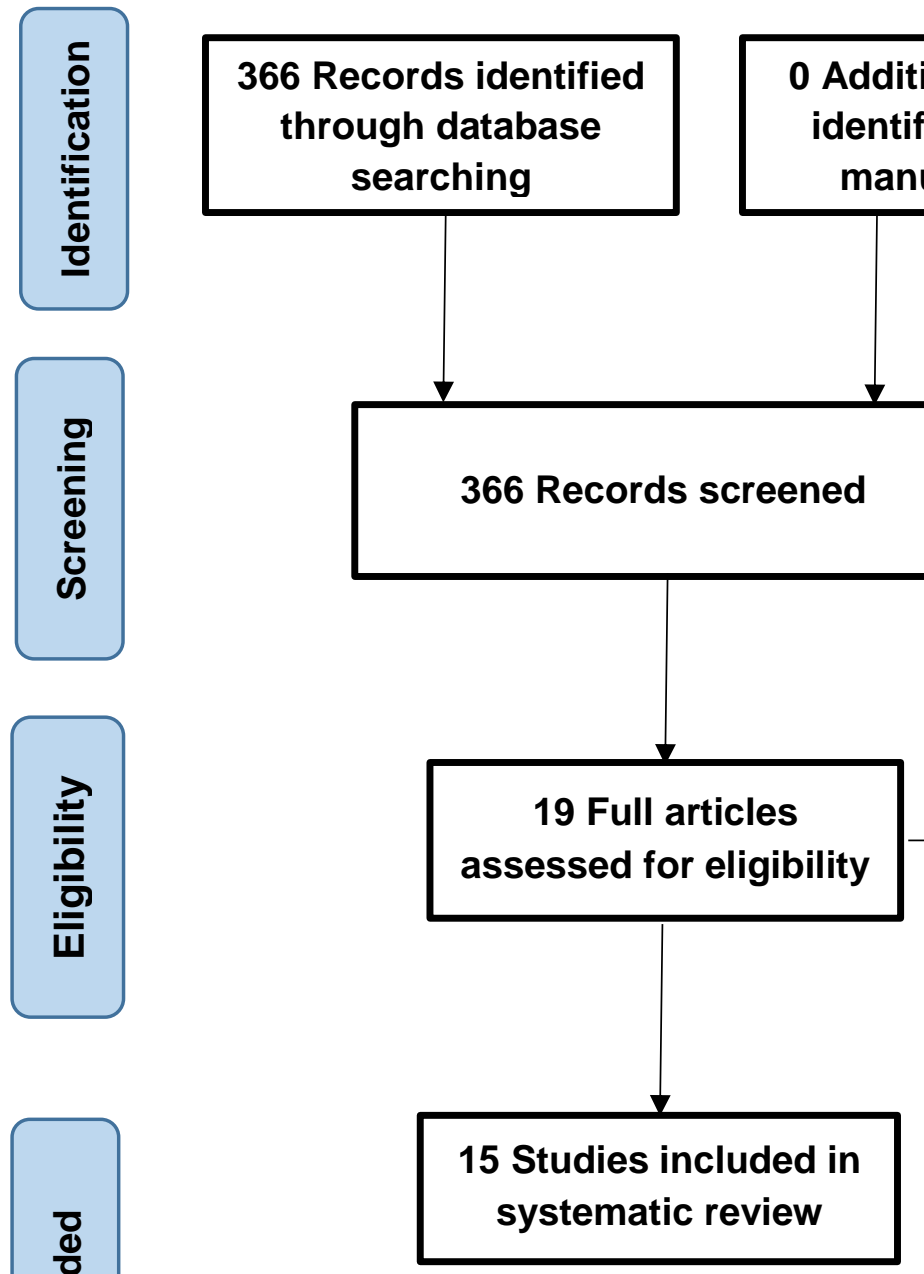

347 Studies excluded as deemed not relevant

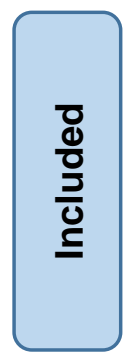

4 studies excluded:

- 1 lack of comparative AMH data

- 1 was a duplication

- 2 including PCOS women 
Table 1. Modified Newcastle Ottawa scale for risk of bias and quality assessment of the included studies

\begin{tabular}{|c|c|c|c|c|c|}
\hline Author & Year & $\begin{array}{c}\text { Selection } \\
(* * *)\end{array}$ & $\begin{array}{c}\text { Comparability } \\
(* * * *)\end{array}$ & $\begin{array}{c}\text { Outcome } \\
(* *)\end{array}$ & Overall \\
\hline \multicolumn{6}{|l|}{ Short-term studies } \\
\hline Arbo et al. (14) & 2007 & $*$ & $* * * *$ & - & 5 \\
\hline Streuli et al. (24) & 2008 & $*$ & $* * * *$ & $*$ & 6 \\
\hline Andersen et al. (34) & 2011 & $* *$ & $* * *$ & - & 5 \\
\hline \multicolumn{6}{|l|}{ Medium-term studies } \\
\hline Somunkiran et al. (25) & 2007 & - & $* * * *$ & $* *$ & 6 \\
\hline Li et al. (27) & 2011 & $* *$ & $* * * *$ & $* *$ & 8 \\
\hline Kallio et al. (21) & 2013 & $* *$ & $* * * *$ & $*$ & 7 \\
\hline \multicolumn{6}{|l|}{ Long-term studies } \\
\hline Deb et al. (29) & 2012 & $*$ & $* * *$ & $* *$ & 6 \\
\hline Bentzen et al. (19) & 2012 & $* *$ & $* * *$ & - & 5 \\
\hline Dolleman et al. (22) & 2013 & $* *$ & - & - & 2 \\
\hline Petersen et al. (24) & 2015 & $* *$ & $* * *$ & - & 5 \\
\hline \multicolumn{6}{|l|}{ Varied-term studies } \\
\hline Shaw et al. (18) & 2011 & $*$ & - & $*$ & 2 \\
\hline Kristensen et al. (20) & 2012 & $*$ & $* * *$ & - & 4 \\
\hline Johnson et al. (23) & 2014 & $*$ & $*$ & $* *$ & 4 \\
\hline \multicolumn{6}{|l|}{ Previous HC users } \\
\hline Kucera et al. (37) & 2016 & $* *$ & $* * * *$ & $* *$ & 8 \\
\hline Van den Berg et al. (38) & 2010 & $*$ & $* * *$ & - & 4 \\
\hline
\end{tabular}


Table 2 characteristics of eight short- and medium-term studies comparing AMH before and during CHC

\begin{tabular}{|c|c|c|c|c|c|c|c|c|c|c|c|}
\hline \multirow{2}{*}{ Author } & \multirow{2}{*}{ Country } & \multirow{2}{*}{ Design } & \multirow{2}{*}{$\mathrm{n}$} & \multicolumn{4}{|c|}{ Contraception } & \multirow{2}{*}{$\begin{array}{c}\text { Age } \\
\text { (years)* }\end{array}$} & \multicolumn{2}{|c|}{ AMH } & \multirow{2}{*}{$\begin{array}{l}\text { Secondary } \\
\text { Outcomes }\end{array}$} \\
\hline & & & & Pattern & Duration & Route & $\mathrm{n}$ & & Outcome & Kit & \\
\hline \multicolumn{12}{|l|}{ Short-term studies (3 weeks) } \\
\hline Arbo et al, 2007 (16) & Brazil & Cohort & 20 & Mid-luteal & 3 weeks & Oral $^{1}$ & 20 & $29.1 \pm 4.1$ & $\downarrow$ & DSL & $\mathrm{FSH}, \mathrm{AFC}, \mathrm{E}_{2}$ \\
\hline \multirow[b]{2}{*}{ Streuli et al, 2008 (26) } & \multirow[b]{2}{*}{ Switzerland } & \multirow[b]{2}{*}{$\mathrm{RCT}$} & \multirow[b]{2}{*}{14} & \multirow[b]{2}{*}{ Cyclical } & \multirow[b]{2}{*}{3 weeks } & Oral $^{1}$ & 7 & \multirow[b]{2}{*}{$24.1 \pm 3.5$} & \multirow[b]{2}{*}{ No change } & \multirow[b]{2}{*}{ IOT } & \multirow[b]{2}{*}{$\mathrm{FSH}, \mathrm{AFC}$} \\
\hline & & & & & & Vaginal $^{5}$ & 7 & & & & \\
\hline Andersen et al, 2011 (36) & $\mathrm{USA}^{* *}$ & $\mathrm{RCT}$ & 209 & Cyclical & 3 weeks & Oral $^{1}$ & 209 & $31.8 \pm 3.7$ & No change & DSL & $\mathrm{AFC}, \mathrm{FSH}, \mathrm{E}_{2}$ \\
\hline \multicolumn{12}{|c|}{ Medium-term studies (2 - 6 months) } \\
\hline Somunkiran et al, 2007 (25) & Turkey & Cohort & 15 & Cyclical & 6 months & $\mathrm{Oral}^{2}$ & & $25.1 \pm 6.9$ & No change & DSL & $\mathrm{FSH}, \mathrm{AFC}, \mathrm{OV}$ \\
\hline Li et al, 2011 (27) & China & Cohort & 46 & Cyclical & 4 months & $\begin{array}{l}\text { Oral }^{3} \\
\text { Injectable }^{6}\end{array}$ & $\begin{array}{l}23 \\
23\end{array}$ & $\begin{array}{l}30[26,35] \\
32[26,35]\end{array}$ & No change & IOT & - \\
\hline \multirow{3}{*}{ Kallio et al, 2013 (21) } & \multirow{3}{*}{ Finland } & \multirow{3}{*}{$\mathrm{RCT}$} & \multirow{3}{*}{42} & \multirow{3}{*}{ Continuous } & \multirow{3}{*}{9 weeks } & Oral $^{1}$ & 13 & $23.5 \pm 3.1$ & \multirow{3}{*}{$\downarrow$} & \multirow{3}{*}{ Gen II } & \\
\hline & & & & & & Transdermal $^{7}$ & 15 & $24.3 \pm 3.5$ & & & FSH, $\mathrm{E}_{2}$ \\
\hline & & & & & & Vaginal $^{5}$ & 14 & $24.1 \pm 3.7$ & & & \\
\hline
\end{tabular}

* Age presented as mean $\pm \mathrm{SD}$; median [interquartile]; mean $( \pm \mathrm{SEM})$

** Multicentre trial in different countries: USA, Denmark, Germany, Spain, Turkey

${ }^{1} \mathrm{EE}+$ desogestrel,

${ }^{2} \mathrm{EE}+$ cyproterone acetate,

3 ethinylestradiol + levonorgestrel,

4 ethinylestradiol + drospirenone

${ }^{5} \mathrm{EE}+$ etonogestrel

${ }^{6}$ Estradiol cypionate + Medroxyprogesterone acetate

${ }^{7} \mathrm{EE}+$ norelgestromin

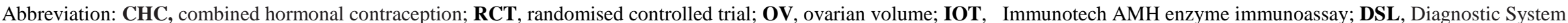

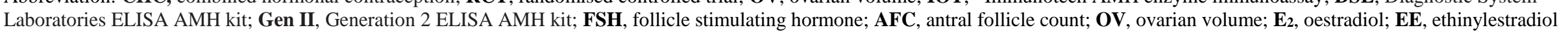


Table 3 Characteristics of eight Cross-sectional studies comparing AMH between Long- ( $>1$ year) and varied-term users versus non-users of HC

\begin{tabular}{|c|c|c|c|c|c|c|c|c|c|c|c|c|}
\hline \multirow[b]{2}{*}{ Author } & \multirow[b]{2}{*}{ Country } & \multicolumn{2}{|c|}{$\mathrm{n}$} & \multicolumn{4}{|c|}{ Contraception } & \multicolumn{2}{|c|}{ Age $($ years) $*$} & \multicolumn{2}{|l|}{$\mathrm{AMH}$} & \multirow{2}{*}{$\begin{array}{l}\text { Secondary } \\
\text { Outcomes }\end{array}$} \\
\hline & & Users & $\begin{array}{l}\text { Non- } \\
\text { users }\end{array}$ & $\begin{array}{c}\text { Duration } \\
\text { (Years) }\end{array}$ & Type & Route & $\mathrm{n}$ & Users & Non-users & Outcome & Kit & \\
\hline \multicolumn{13}{|c|}{ Studies assessing AMH in current long-term HC users versus non-users } \\
\hline Deb et al, 2012 (29) & UK & 34 & 36 & $\begin{array}{l}3.2 * * * \\
(2.4-6.0)\end{array}$ & $\mathrm{CHC}$ & Oral $^{3}$ & 34 & $25.4 \pm 4.2$ & $27.2 \pm 4.6$ & No difference & DSL & $\begin{array}{l}\text { FSH, AFC, } \\
\text { OV }\end{array}$ \\
\hline Bentzen et al, 2012 (19) & Denmark & 228 & 504 & NS & $\mathrm{CHC}$ & $\begin{array}{l}\text { Oral }^{8} \\
\text { Vaginal }^{9}\end{array}$ & $\begin{array}{l}217 \\
11\end{array}$ & $\begin{array}{l}29.2 \\
{[26.9,32.6]}\end{array}$ & $\begin{array}{l}33.7 \\
{[30.9,36.9]}\end{array}$ & $\downarrow(30 \%)$ & IOT & $\mathrm{AFC}$ \\
\hline Dolleman et al, 2013 (22) & Netherlands & 927 & 1418 & $>1$ & NS & $\mathrm{Oral}^{9}$ & 927 & \multicolumn{2}{|c|}{$37.3 \pm 9.2^{\pi}$} & $\downarrow$ (11 percentile) & Gen II & - \\
\hline Petersen et al, 2015 (24) & Demark & 244 & 643 & $\begin{array}{l}12 * * \\
(4.0,18.4)\end{array}$ & $\mathrm{CHC}$ & $\begin{array}{l}\text { Oral }^{8} \\
\text { Vaginal }^{9}\end{array}$ & $\begin{array}{l}225 \\
19\end{array}$ & $31.5 \pm 4.3$ & $34.1 \pm 4.3$ & $\downarrow(19 \%)$ & IOT & $\mathrm{AFC}, \mathrm{OV}$ \\
\hline \multicolumn{13}{|c|}{ Studies assessing AMH in current varied-term HC users versus non-users } \\
\hline Shaw et al, 2011 (18) & USA & 14 & 121 & NS & NS & Oral $^{9}$ & 14 & \multicolumn{2}{|c|}{$41 \pm 2.48^{\pi}$} & $\downarrow(35 \%)$ & DSL & - \\
\hline Johnson et al, 2014 (23) & USA & 8 & 115 & NS & $\begin{array}{l}\mathrm{CHC} \\
\mathrm{POC}\end{array}$ & $\begin{array}{l}\text { Oral }^{9} \\
\text { Vaginal }^{9}\end{array}$ & $?$ & $\begin{array}{l}28.5 \\
{[25.5,30.0]}\end{array}$ & $\begin{array}{l}26.0 \\
{[22.0,29.0]}\end{array}$ & $\downarrow(55 \%)$ & Gen II & $\mathrm{AFC}$ \\
\hline \multicolumn{13}{|c|}{ Studies assessing AMH in previous long-term $\mathrm{HC}$ users versus non-users } \\
\hline Dolleman et al, 2013 (22) & Netherlands & 1194 & 1418 & NS & NS & Oral $^{9}$ & 1194 & \multicolumn{2}{|c|}{$37.3 \pm 9.2^{\pi}$} & No difference & Gen II & - \\
\hline Kucera et al, 2016 (37) & $\begin{array}{l}\text { Czech } \\
\text { Rep. }\end{array}$ & 105 & 44 & $\begin{array}{l}11.4^{\pi \pi} \\
(10.0-17.0)\end{array}$ & $\mathrm{CHC}$ & Oral $^{9}$ & 105 & $32.1(27-34)$ & $31.2(23-34)$ & No difference & Access & - \\
\hline
\end{tabular}

* Age presented as mean $\pm \mathrm{SD}$, median [interquartile], mean (range),

** median ( $90 \%$ population limit)

$* * *$ average (range)

$\pi \pi$ mean (range)

${ }^{\pi}$ Age of all women in both groups (study and control)

${ }^{\delta \delta}$ intra-uterine device, 1 ; injection, 1

3 ethinylestradiol + levonorgestrel,

${ }^{8} \mathrm{EE}+$ unspecified progestogen

${ }^{9}$ Unspecified hormonal components 


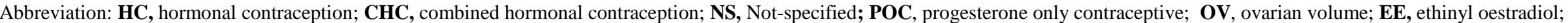

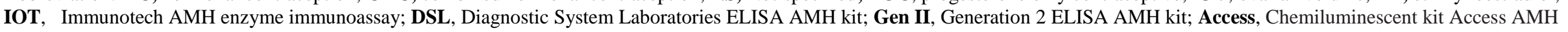
(Beckman Coulter, Brea, CA); FSH, follicle stimulating hormone; AFC, antral follicle count; E2, oestradiol; 